Vietnam Journal of Mechanics, VAST, Vol. 34, No. 3 (2012), pp. $157-167$

\title{
CALCULATION OF NONLINEAR VIBRATIONS OF PIECEWISE-LINEAR SYSTEMS USING THE SHOOTING METHOD
}

\author{
Nguyen Van Khang, Hoang Manh Cuong, Nguyen Thai Minh Tuan \\ Hanoi University of Science and Technology, Vietnam
}

\begin{abstract}
In this paper, an explicit formulation of the shooting scheme for computation of multiple periodic attractors of a harmonically excited oscillator which is asymmetric with both stiffness and viscous damping piecewise linearities is derived. The numerical simulation by the shooting method is compared with that by the incremental harmonic balance method (IHB method), which shows that the shooting method is in many respects distinctively advantageous over the incremental harmonic balance method.
\end{abstract}

Key words: Nonlinear vibration, shooting method, numerical simulation, piecewise linear system, Floquet stability.

\section{INTRODUCTION}

One of the most important objectives of the nonlinear dynamics is to determine the periods of the periodic orbits of the strongly nonlinear dynamic systems, since it is related to many important problems such as bifurcation, stability, chaos and so on. There are many ways to determine periodic orbits and periods of a nonlinear dynamic system. Those methods generally can be classified into categories, namely, frequency domain methods and time domain methods. In the frequency-domain formulation, the method of harmonic balance and generalized versions of this method are commonly used. In the time-domain formulation, finite-difference schemes, shooting techniques, and Poincare map methods are also widely used [1-5].

In recent years, much attention has been paid to the system with piecewise-linearity. The considered oscillator is a typical nonlinear system that is widely used in engineering systems. The determination of the periodic vibrations of piecewise-linear systems using the incremental harmonic balance method has been studied by a number of papers [6-10]. In the present paper, the vibrations of a system subjected to harmonic excitation, which includes both stiffness and viscous damping piecewise linearities, is investigated using the shooting method. 


\section{AN OVERVIEW OF THE SHOOTING METHOD}

We consider now nonautonomous systems defined by

$$
\dot{\boldsymbol{x}}=\boldsymbol{f}(\boldsymbol{x}, t, \boldsymbol{\mu}),
$$

where $\boldsymbol{x} \in \mathrm{R}^{n}, \boldsymbol{f} \in \mathrm{R}^{n}, \boldsymbol{\mu} \in \mathrm{R}^{m}$ and $\boldsymbol{f}(\boldsymbol{x}, t, \boldsymbol{\mu})$ is a periodic function with period $T_{e}$

$$
\boldsymbol{f}\left(\boldsymbol{x}, t+T_{e}, \boldsymbol{\mu}\right)=\boldsymbol{f}(\boldsymbol{x}, t, \boldsymbol{\mu}) .
$$

A solution $\boldsymbol{x}=\boldsymbol{\phi}(t)$ of a continuous-time system is periodic with the period $T$ if $\phi(t+T)=$ $\phi(t)$ and $\phi(t+\tau) \neq \phi(t)$ for $0<\tau<T$.

In this section, we describe the shooting method for finding periodic solutions $T=$ $n T_{e}$ of the nonautonomous systems (1). Suppose that a periodic solution of the system (1) exists and its period is $T$. This solution must be satisfied the following condition

$$
\boldsymbol{x}(0)=\boldsymbol{x}(T) .
$$

According to the shooting method, the initial - value problem

$$
\dot{\boldsymbol{x}}=\boldsymbol{f}(\boldsymbol{x}, t, \boldsymbol{\mu}), \quad \boldsymbol{x}(0)=\boldsymbol{x}_{0}=\boldsymbol{\eta},
$$

is converted into a two - point boundary - value problem

$$
\dot{\boldsymbol{x}}=\boldsymbol{f}(\boldsymbol{x}, t, \boldsymbol{\mu}), \quad \boldsymbol{x}(T, \boldsymbol{\eta})=\boldsymbol{x}(0)=\boldsymbol{\eta} .
$$

Thus, one seeks an initial condition $\boldsymbol{x}(0)=\boldsymbol{\eta}$ and a solution $\boldsymbol{x}(t, \boldsymbol{\eta})$ with a minimal period $T$ such that

$$
\boldsymbol{x}(T, \boldsymbol{\eta})-\boldsymbol{\eta}=\mathbf{0}
$$

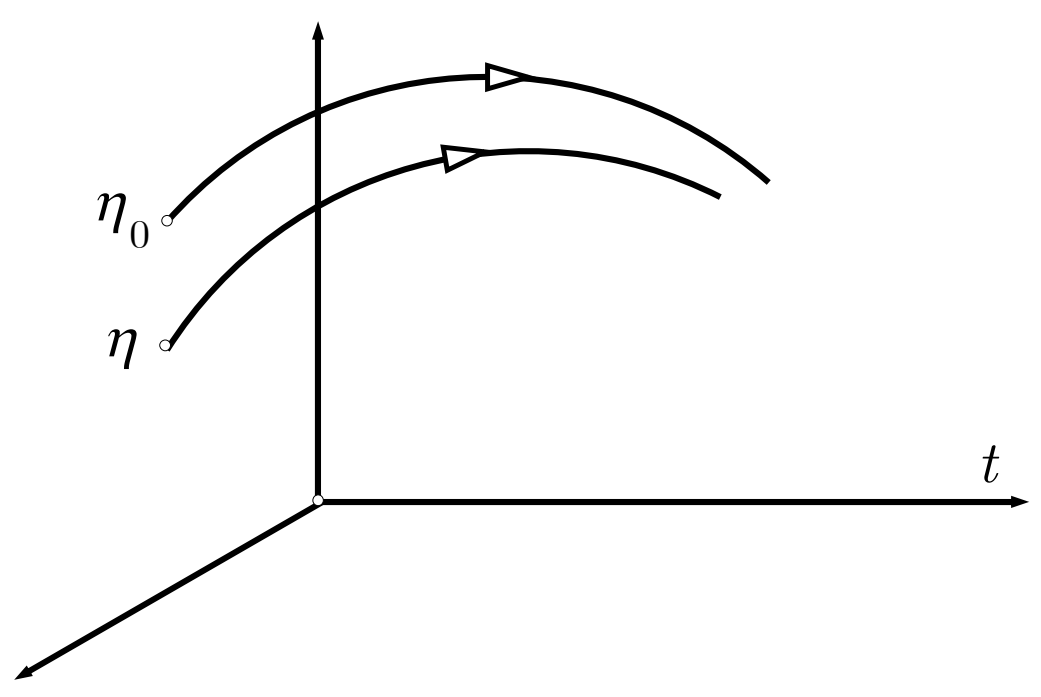

Fig. 1. Depiction of the shooting method for a two dimensional system 
In Fig. 1, we graphically illustrate the shooting method when Eq. (1) is two - dimensional. The trajectory running from $\boldsymbol{\eta}$ at $t=0$ to the same location at $t=T$ represents the desired periodic solution. The other trajectory represents the solution obtained by using the initial guess $\boldsymbol{\eta}^{(0)}$ for $\boldsymbol{\eta}$. Because this initial guess is off the mark, it needs correction. The correction is accomplished through a Newton - Raphson scheme as described below.

We seek $\delta \boldsymbol{\eta}=\boldsymbol{\eta}-\boldsymbol{\eta}^{(0)}$, such that Eq. (6) is satisfied within a specified tolerance. That is

$$
\boldsymbol{x}\left(T, \boldsymbol{\eta}^{(0)}+\delta \boldsymbol{\eta}\right)-\left(\boldsymbol{\eta}^{(0)}+\delta \boldsymbol{\eta}\right) \approx 0 .
$$

Expanding $\boldsymbol{x}\left(T, \boldsymbol{\eta}^{(0)}+\delta \boldsymbol{\eta}\right)$ in a Taylor series and keeping only linear terms in $\delta \boldsymbol{\eta}$, we obtain

$$
\boldsymbol{x}\left(T, \boldsymbol{\eta}^{(0)}+\delta \boldsymbol{\eta}\right) \approx \boldsymbol{x}\left(T, \boldsymbol{\eta}^{(0)}\right)+\frac{\partial \boldsymbol{x}}{\partial \boldsymbol{\eta}}\left(T, \boldsymbol{\eta}^{(0)}\right) \delta \boldsymbol{\eta} .
$$

Substitution of Eq. (8) into Eq. (7) yields

$$
\boldsymbol{x}\left(T, \boldsymbol{\eta}^{(0)}\right)+\frac{\partial \boldsymbol{x}}{\partial \boldsymbol{\eta}}\left(T, d \boldsymbol{\eta}^{(0)}\right) \delta \boldsymbol{\eta}=\boldsymbol{\eta}^{(0)}+\delta \boldsymbol{\eta}
$$

From Eq. (9) it follows

$$
\left[\frac{\partial \boldsymbol{x}}{\partial \boldsymbol{\eta}}\left(T, \boldsymbol{\eta}^{(0)}\right)-\boldsymbol{I}\right] \delta \boldsymbol{\eta}=\boldsymbol{\eta}^{(0)}-\boldsymbol{x}\left(T, \boldsymbol{\eta}^{(0)}\right)
$$

where $\boldsymbol{I}$ denotes the $\mathrm{n} \times \mathrm{n}$ identity matrix, $\partial \boldsymbol{x} / \partial \boldsymbol{\eta}$ is a $\mathrm{n} \times \mathrm{n}$ matrix, and $\boldsymbol{x}\left(T, \boldsymbol{\eta}^{(0)}\right)$ is a $\mathrm{n} \times 1$ vector. Vector $\boldsymbol{x}\left(T, \boldsymbol{\eta}^{(0)}\right)$ is determined by solving Eq. (4) with the initial condition $\boldsymbol{x}(0)=\boldsymbol{\eta}^{(0)}$.

Differentiating both sides of Eq. (1) with respect to $\boldsymbol{\eta}$, we obtain

$$
\frac{d}{d t}\left(\frac{\partial \boldsymbol{x}}{\partial \boldsymbol{\eta}}\right)=\frac{\partial}{\partial \boldsymbol{\eta}}\left(\frac{d \boldsymbol{x}}{d t}\right)=\frac{\partial \boldsymbol{f}(\boldsymbol{x}, t, \boldsymbol{\mu})}{\partial \boldsymbol{\eta}}=\frac{\partial \boldsymbol{f}(\boldsymbol{x}, t, \boldsymbol{\mu})}{\partial \boldsymbol{x}} \frac{\partial \boldsymbol{x}}{\partial \boldsymbol{\eta}} .
$$

Using the following definition of the Jacobian matrix

$$
\frac{\partial \boldsymbol{f}}{\partial \boldsymbol{x}}=\left[\begin{array}{ccc}
\partial f_{1} / \partial x_{1} & \ldots & \partial f_{1} / \partial x_{n} \\
\ldots & \ldots & \ldots \\
\partial f_{n} / \partial x_{1} & \ldots & \partial f_{n} / \partial x_{n}
\end{array}\right]=D_{\boldsymbol{x}} \boldsymbol{f}(\boldsymbol{x}, t, \boldsymbol{\mu}) .
$$

Eq. (11) can be rewritten in following form

$$
\frac{d}{d t}\left(\frac{\partial \boldsymbol{x}}{\partial \boldsymbol{\eta}}\right)=D_{\boldsymbol{x}} \boldsymbol{f}(\boldsymbol{x}, t, \boldsymbol{\mu}) \frac{\partial \boldsymbol{x}}{\partial \boldsymbol{\eta}}
$$

Also, the differentiation of the initial condition $\boldsymbol{x}(0)=\boldsymbol{\eta}$ with respect to $\boldsymbol{\eta}$ leads to

$$
\frac{\partial \boldsymbol{x}(0)}{\partial \boldsymbol{\eta}}=\boldsymbol{I}
$$

The components of the $\mathrm{n} \times \mathrm{n}$ matrix $\partial \boldsymbol{x} / \partial \boldsymbol{\eta}$ evaluated at $\left(\boldsymbol{\eta}^{(0)}, T\right)$ need to be determined. After $\boldsymbol{x}$ is determined, we can solve the linear initial-value problem (13) and (14) from zero to $\mathrm{T}$ and evaluate $\partial \boldsymbol{x} / \partial \boldsymbol{\eta}$ at $\left(\boldsymbol{\eta}^{(0)}, T\right)$. Instead of solving Eq. (4) subjected to $\boldsymbol{x}(0)=\boldsymbol{\eta}^{(0)}$, saving the solution $\boldsymbol{x}(t)$ for $0<\tau<T$ and then solving Eq. (13) and Eq. 
(14), we can solve both problems simultaneously, thereby obtaining $\boldsymbol{x}\left(T, \boldsymbol{\eta}^{(0)}\right)$ and $\partial \boldsymbol{x} / \partial \boldsymbol{\eta}$ at $\left(\boldsymbol{\eta}^{(0)}, T\right)$ simultaneously.

Once the matrix $\partial \boldsymbol{x} / \partial \boldsymbol{\eta}$ evaluated at $\left(\boldsymbol{\eta}^{(0)}, T\right)$ is known, we have a system of $n$ algebraic equation, namely, Eq. (10), to determine $n$ unknowns $\delta \boldsymbol{\eta}$. Then, the initial guess $\boldsymbol{\eta}^{(0)}$ is updated, and the procedure is repeated until the specified convergence criterion is satisfied.

\section{AN OVERVIEW OF STABILITY OF THE COMPUTED PERIODIC SOLUTION}

We let a periodic solution of Eq. (1) at $\boldsymbol{\mu}=\boldsymbol{\mu}^{*}$ be denoted by $\boldsymbol{x}^{*}(t)$ having the minimal period $T$. Then, a disturbance $\boldsymbol{y}(t)$ is superimposed on $\boldsymbol{x}^{*}(t)$, resulting in

$$
\boldsymbol{x}(t)=\boldsymbol{x}^{*}(t)+\boldsymbol{y}(t)
$$

Substituting Eq. (15) into Eq. (1), assuming that $\boldsymbol{f}$ is at least twice continuously differentiable, and expanding the obtained result in a Taylor series about $\boldsymbol{x}^{*}(t)$, we obtain

$$
\dot{\boldsymbol{x}}^{*}(t)+\dot{\boldsymbol{y}}(t)=\boldsymbol{f}\left(\boldsymbol{x}^{*}+\boldsymbol{y}, t, \boldsymbol{\mu}^{*}\right)+\left.\frac{\partial \boldsymbol{f}}{\partial \boldsymbol{x}}\right|_{\boldsymbol{x}^{*}} \boldsymbol{y}+\ldots
$$

Retaining only linear terms in the disturbance, from Eq. (16) it follows

$$
\dot{\boldsymbol{y}}=D_{\boldsymbol{x}} \boldsymbol{f}\left(\boldsymbol{x}^{*}, t, \boldsymbol{\mu}^{*}\right) \boldsymbol{y}=\boldsymbol{P}(t) \boldsymbol{y}
$$

where

$$
D_{\boldsymbol{x}} \boldsymbol{f}\left(\boldsymbol{x}^{*}, t, \boldsymbol{\mu}^{*}\right)=\left.\frac{\partial \boldsymbol{f}(\boldsymbol{x}, t, \boldsymbol{\mu})}{\partial \boldsymbol{x}}\right|_{\boldsymbol{x}^{*}}=\boldsymbol{P}(t) .
$$

Note that matrix $\boldsymbol{P}(t)$ is time - periodic with period $T$.

Thus, we shall consider a system of homogeneous differential equations

$$
\dot{\boldsymbol{x}}=\boldsymbol{P}(t) \boldsymbol{x},
$$

where $\boldsymbol{P}(t)$ is a continuous $T$ - periodic $\mathrm{n} \times \mathrm{n}$ matrix. The $\mathrm{n}-$ dimensional linear system (19) has $n$ linearly independent solutions $\boldsymbol{y}_{i}$, where $i=1, \ldots, n$. These solutions are usually called a fundamental set of solutions of the system (17). According to Floquet theory [11 16], the characteristic equation of Eq. (19) is independent of the chosen fundamental set of solutions. Therefore, the characteristic equation can be formulated by the following way. Firstly, we specify a set of $n$ initial conditions $\boldsymbol{x}_{i}(0)$ for $i=1, \ldots, n$, their elements

$$
x_{s}^{(i)}(0)= \begin{cases}1 & \text { when } s=i \\ 0 & \text { otherwhile }\end{cases}
$$

and $\left[\boldsymbol{x}_{1}(0), \boldsymbol{x}_{2}(0), \ldots, \boldsymbol{x}_{n}(0)\right]=\boldsymbol{I}$, where $\boldsymbol{I}$ denotes the $\mathrm{n} \times \mathrm{n}$ identity matrix. By implementing numerical integration of Eq. (19) within interval $[0, T]$ for $n$ given initial conditions respectively, we obtain $n$ vectors $\boldsymbol{x}_{i}(T), i=1, \ldots, n$. The matrix $\boldsymbol{\Phi}(t)$ defined by

$$
\boldsymbol{\Phi}(T)=\left[\boldsymbol{x}_{1}(T), \boldsymbol{x}_{2}(T), \ldots, \boldsymbol{x}_{n}(T)\right]
$$


is called the monodromy matrix [5 - 6] of Eq. (19). The characteristic equation of Eq. (19) can then be written in the form

$$
|\boldsymbol{\Phi}(T)-\rho \boldsymbol{I}|=\left|\begin{array}{cccc}
x_{1}^{(1)}(T)-\rho & x_{2}^{(1)}(T) & \ldots & x_{n}^{(1)}(T) \\
x_{1}^{(2)}(T) & x_{2}^{(2)}(T)-\rho & \ldots & x_{n}^{(2)}(T) \\
\ldots & \ldots \ldots & \ldots . & \ldots . \\
x_{1}^{(n)}(T) & x_{2}^{(n)}(T) & \ldots . & x_{n}^{(n)}(T)-\rho
\end{array}\right|=0
$$

Expansion of Eq. (22) yields a $n$ - order algebraic equation

$$
\rho^{n}+a_{1} \rho^{n-1}+a_{2} \rho^{n-2}+\ldots .+a_{n-1} \rho+a_{n}=0,
$$

where unknowns $\rho_{i}(i=1, \ldots, n)$, called Floquet multipliers, can be determined from Eq. (23). Floquet exponents are given by

$$
\lambda_{k}=\frac{1}{T} \ln \rho_{k}, \quad(k=1, \ldots, n) .
$$

When the Floquet multipliers or Floquet exponents are known, the stability conditions of periodic solutions of the system of nonlinear differential equations (1) can be easily determined using the Floquet theorem [11 - 14].

\section{PERIODIC NONLINEAR VIBRATIONS OF PIECEWISE-LINEAR SYSTEM}

The nonlinear oscillator considered here is a single - degree - of - freedom vibration system with both stiffness and viscous damping piecewise linearities as shown in Fig. 2, which serves as a model for a wide range of piecewise-linear vibration systems in engineering practice. Complex dynamic properties such as the static bifurcation and periodic doubling bifurcation to chaos of the system have been studied in Ref. [9] using the theory of singularity, Runge-Kutta numerical integration method and Poincare map. Using the IHB method, one has been calculated the period- 2 solution, the period - 3 solution, the period - 4 solution and the period - 8 solution of this system $[6,7]$.

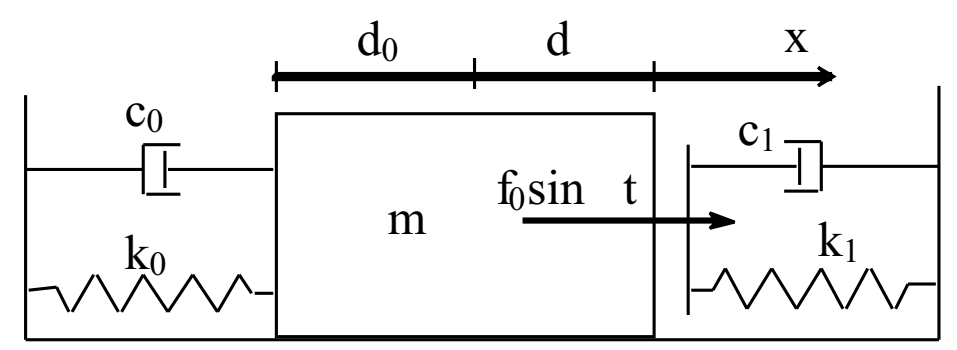

Fig. 2. Vibration model of a piecewise - linear system

Suppose that without the mass $m$, the two free springs would just touch each other. For the damper and the linear spring which are at the left side of the mass and connected to it, and those at the right side and not connected to it, their respective damping or 
stiffness are denoted by $c_{0}, k_{0}, c_{1}$ and $k_{1}$. Assume that when the oscillator is at the state of static equilibrium, $d_{0}$ and $d$, respectively, denote the compression of the spring at both sides and thus $k_{0} d_{0}=k_{1} d$. Let the displacement $x$ denote the co-ordinate of the right edge of the mass relative to the fixed point $O$, which itself is the position of the right edge at static equilibrium.

The kinetic energy, potential energy and dissipative function of the considered system can be expressed in the following form

$$
\begin{gathered}
T=\frac{1}{2} m \dot{x}^{2}, \\
\Pi= \begin{cases}\left(k_{0}+k_{1}\right) x^{2} / 2, & x>-d \\
k_{0}\left(x-d_{0}\right)^{2} / 2, & x \leq-d\end{cases} \\
\Phi= \begin{cases}\left(c_{0}+c_{1}\right) \dot{x}^{2} / 2, & x>-d \\
c_{0} \dot{x}^{2} / 2, & x \leq-d\end{cases}
\end{gathered}
$$

Substitution of Eqs. (25) - (27) into the Lagrange equation of the second type

$$
\frac{d}{d t}\left(\frac{\partial T}{\partial \dot{x}}\right)-\frac{\partial T}{\partial x}=-\frac{\partial \Pi}{\partial x}-\frac{\partial \Phi}{\partial \dot{x}}+Q^{*},
$$

yields the differential equation of motion of the system

$$
m \ddot{x}+h(\dot{x})+g(x)=f_{0} \sin \Omega t
$$

where

$$
h(\dot{x})=\left\{\begin{array}{ll}
\left(c_{0}+c_{1}\right) \dot{x}, & x>-d \\
c_{0} \dot{x}, & x \leq-d
\end{array} ; \quad g(x)= \begin{cases}\left(k_{0}+k_{1}\right) x, & x>-d \\
k_{0}\left(x-d_{0}\right), & x \leq-d\end{cases}\right.
$$

Functions $h(\dot{x})$ and $g(x)$ can be rewritten in the following form

$$
h(\dot{x})=c_{0}[\dot{x}+H(\dot{x})], \quad g(x)=k_{0} x+G(x),
$$

where

$$
H(\dot{x})=\left\{\begin{array}{ll}
c_{1} \dot{x} / c_{0}, & x>-d \\
0, & x \leq-d
\end{array} ; \quad G(x)= \begin{cases}k_{1} x, & x>-d \\
-k_{0} d_{0}, & x \leq-d\end{cases}\right.
$$

From this it follows the differential equation of the piecewise - linear system

$$
m \ddot{x}+c_{0} \dot{x}+k_{0} x+c_{0} H(\dot{x})+G(x)=f_{0} \sin \Omega t .
$$

Eq. (31) can then be expressed in the state space as

$$
\left\{\begin{array}{l}
\dot{x}_{1}=x_{2} \\
\dot{x}_{2}=\left(-c_{0} x_{2}-k_{0} x_{1}-c_{0} H\left(x_{2}\right)-G\left(x_{1}\right)+f_{0} \sin \Omega t\right) / m
\end{array}\right.
$$

where we use state variable $x_{1}=x, x_{2}=\dot{x}$.

As already tested and evaluated in many relevant papers, the shooting method for computing periodic solutions of nonlinear dynamical systems is distinctively advantageous over classical approaches. As to system (32), the control parameter is taken as $k=k_{0} \cdot 10^{-3}$, 
where $k_{0}$ is the stiffness of the linear spring at the left side of the mass, and other parameter values are fixed as

$$
\begin{aligned}
& f_{0}=7,8 \cdot 10^{3}(\mathrm{~N}), m=0,4 \cdot 10^{3}(\mathrm{~kg}), k_{0}=k \cdot 10^{3}(\mathrm{~N} / \mathrm{m}), k_{1}=0,9 \cdot 10^{6}(\mathrm{~N} / \mathrm{m}), \\
& c_{0}=0,05 \cdot 10^{3}(\mathrm{Ns} / \mathrm{m}), c_{1}=0,5 \cdot 10^{3}(\mathrm{Ns} / \mathrm{m}), d=5 \cdot 10^{-3}(\mathrm{~m}), \Omega=34,56(\mathrm{rad} / \mathrm{s}) .
\end{aligned}
$$

It is easily found that when $k$ is very large, which means that the spring at the left side of the mass is quite rigid, the peat amplitude of the steady periodic state of the oscillator will not exceed $d$, thus the system equals to a linear system with period - 1 , corresponding to the period $T_{e}$ of the external harmonic excitation. In the following study, this trivial case will be ignored and $k$ is designated to be relatively small, varying in the range from 3 to 20. Some calculating results are shown in Figs. 3 - 8.

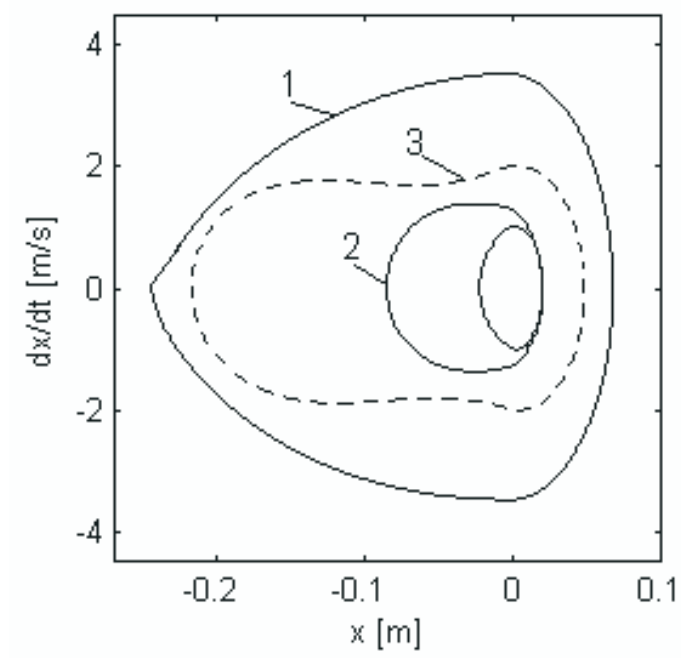

Fig. 3. Phase plane diagrams of multiple periodic response at $k=20$ by the shooting method: (1) stable period-2 motion, (2) stable period - 2 motion, (3) unstable period-2 motion

If we choose $k=20$, we find out three double - period -2 solutions, period $T=$ $2 T_{e}=4 \pi / \Omega$, corresponding to three different initial conditions

$x_{1}(0)=-0,227476 ; x_{2}(0)=0,637536$,

$x_{1}(0)=-0,054747 ; x_{2}(0)=-1,225992$,

$x_{1}(0)=-0,054747 ; x_{2}(0)=-1,225992$.

If we choose $k=6.0$, we find out four period solutions, in there it is a period - 1 solution with $T=T_{e}=2 \pi / \Omega$, two period -2 solutions with $T=2 T_{e}=4 \pi / \Omega$, and a period - 3 solution with $T=3 T_{e}=6 \pi / \Omega$. Using the shooting method we find the following initial conditions of periodic solutions

$$
\begin{aligned}
& x_{1}(0)=-0,023033 ; x_{2}(0)=-1,082459, \\
& x_{1}(0)=-0,144631 ; x_{2}(0)=0,106961 \\
& x_{1}(0)=0,012373 ; x_{2}(0)=-0,433013 \\
& x_{1}(0)=-0,513077 ; x_{2}(0)=-1,247334 .
\end{aligned}
$$




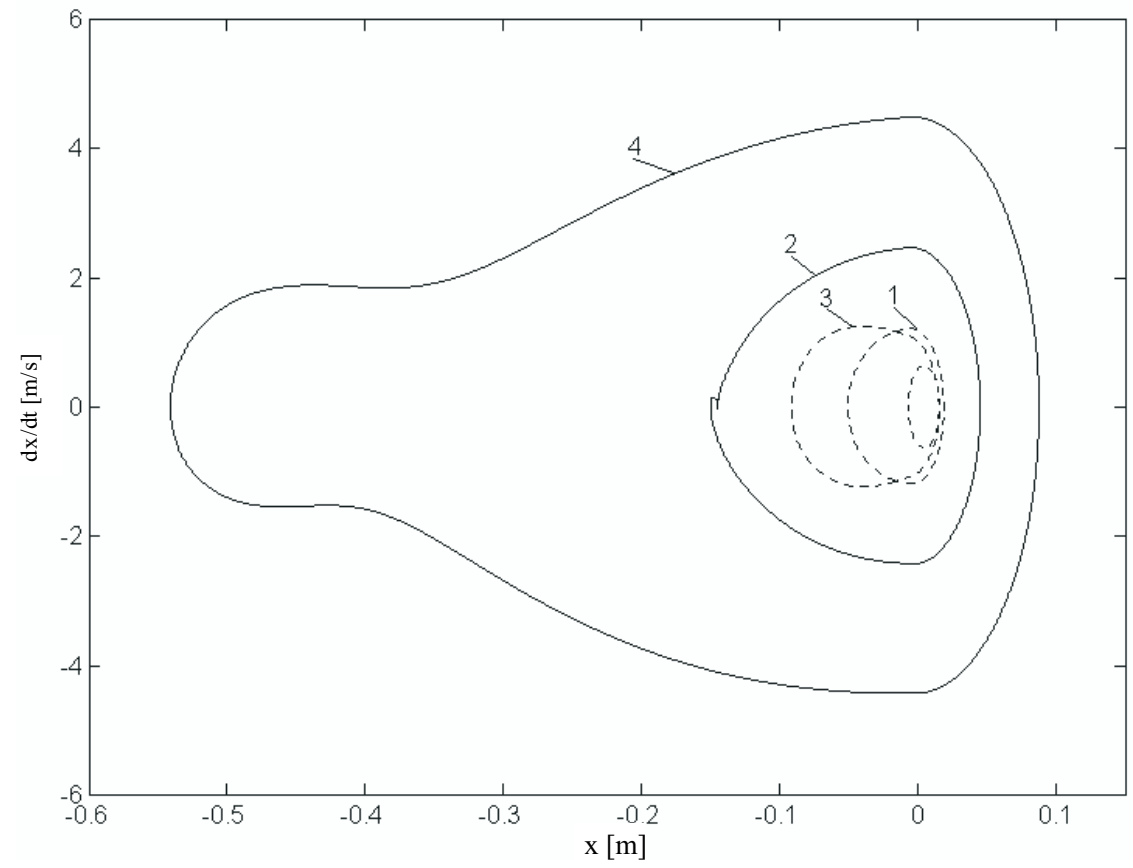

Fig. 4. Phase plane diagrams of multiple periodic response at $k=6$ by the shooting method: (1) unstable period-1 motion, (2) stable period - 2 motion, (3) unstable period - 2 motion, (4) stable period - 3 motion

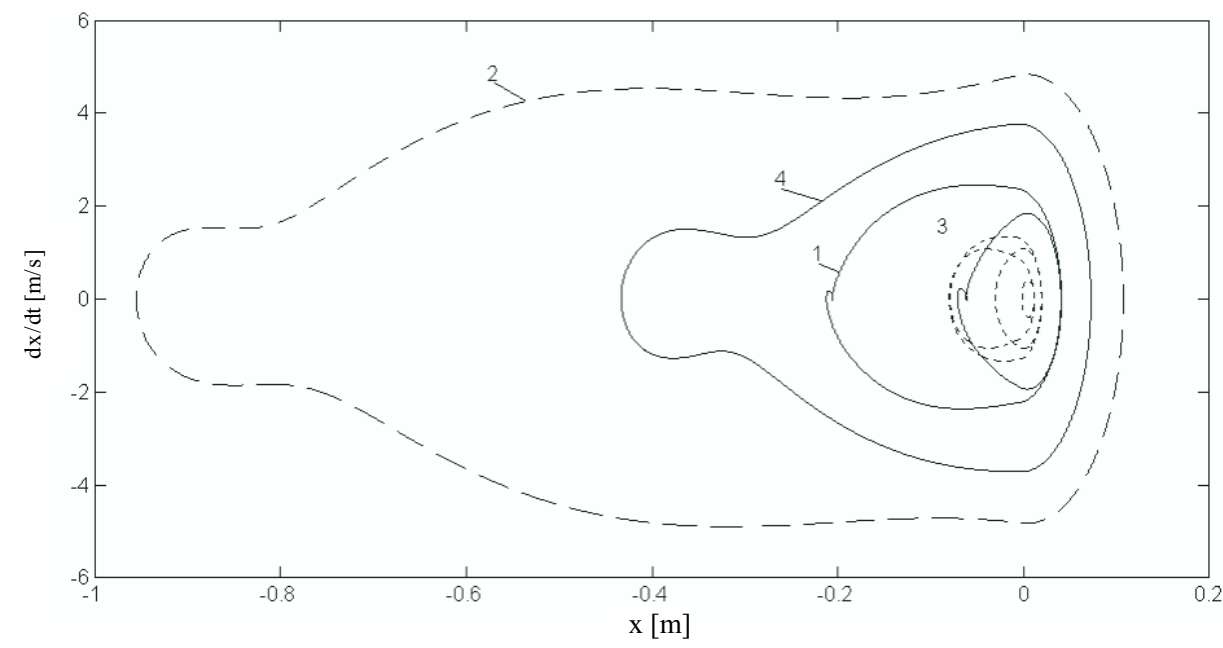

Fig. 5. Phase plane diagrams of multiple periodic response at $k=3.2$ by the shooting method: (1) stable period - 4 motion, (2) unstable period - 4 motion, (3) unstable period - 4 motion, (4) stable period - 3 motion 


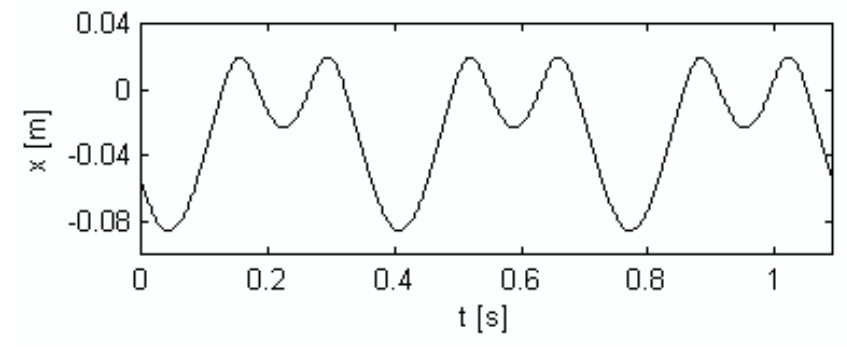

(a)

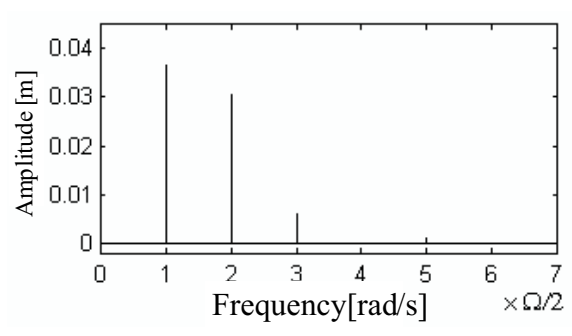

(b)

Fig. 6. The time history and frequency spectrum of the periodic -2 solution at $k=20$, corresponding to initial condition $x_{1}(0)=-0,054747 ; x_{2}(0)=-1,225992$

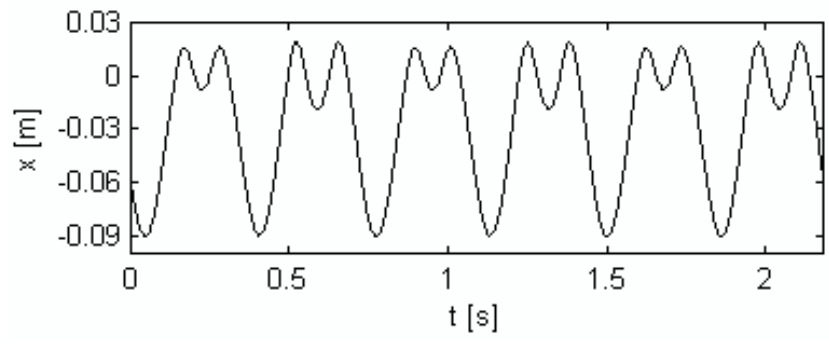

(a)

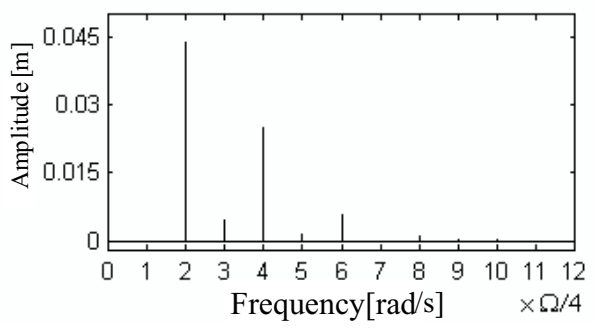

(b)

Fig. 7. The time history and frequency spectrum of the periodic - 4 solution at $k=12$, corresponding to initial condition $x_{1}(0)=-0,057875 ; x_{2}(0)=-1,228845$

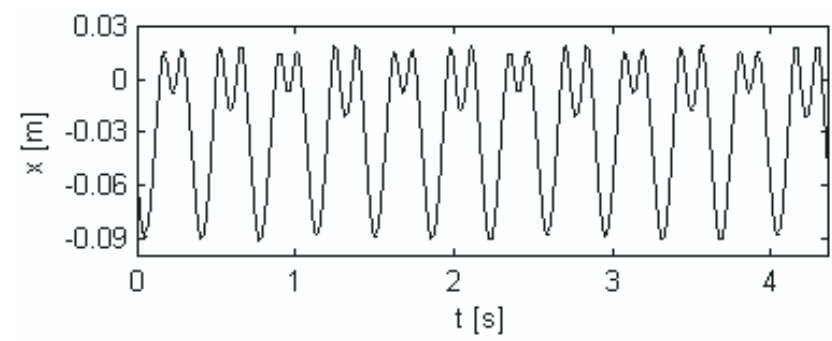

(a)

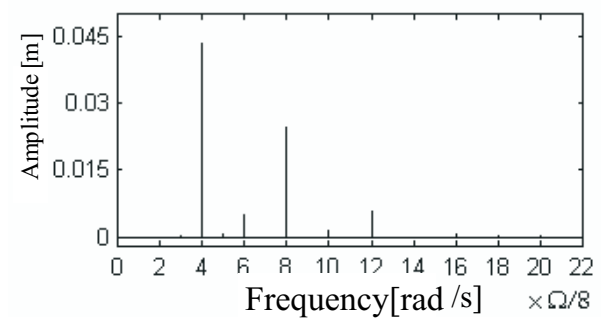

(b)

Fig. 8. The time history and frequency spectrum of the periodic -8 solution at $k=11.63$, corresponding to initial condition $x_{1}(0)=0,055798 ; x_{2}(0)=$ $-1,236303$

If we choose $k=3.2$, we find out four period solutions, in there it is three period - 4 solution with $T=4 T_{e}=8 \pi / \Omega$, a period -3 solution with $T=3 T_{e}=6 \pi / \Omega$, corresponding to four following different initial conditions 
$x_{1}(0)=-0,205468 ; x_{2}(0)=0,094564$,

$x_{1}(0)=-0,103730 ; x_{2}(0)=4,435224$,

$x_{1}(0)=-0,057038 ; x_{2}(0)=-0,947339$,

$x_{1}(0)=-0,407238 ; x_{2}(0)=-1,107569$.

Figs. 3 - 5 show the periodic orbits calculated by the shooting method, corresponding to the above initial conditions.

It is observed from Figs. 3 - 5 that the considered system exhibits the multiple periodic attractors. In the meanwhile, the distinctive phenomenon of coexistence of multiple periodic attractors is observed and analyzed by the interpolated cell mapping method $[17,18]$.

On the other hand, Figs. 6 - 8 show the time histories and frequency spectrums of the displacement $x$, corresponding to the different initial conditions of periodic solutions.

From the above figures, it can be also seen that the considered system performs stable multiple periodic vibrations.

\section{CONCLUSIONS}

In this paper, the shooting method is successfully extended to a class of nonlinear dynamical systems with coexistence of stiffness and viscous damping piecewise linearity for determining periodic solutions. The stability of the periodic solutions is analyzed by using Floquet stability theory. The following concluding remarks have been reached:

- In the theory of linear oscillations we have considered harmonic oscillations only, that is, the oscillation period $T=\frac{2 \pi}{\Omega}$ is the same as that of the external force $f_{0} \sin \Omega t$. However, the oscillations with period of $n T$, where $n$ is an integer, can occur in nonlinear systems subject to a harmonic excitation. These oscillations are called sub-periodic oscillations of $\frac{1}{n}$ order and the corresponding resonance is called the resonance of $n$ kind. This phenomenon appears only in nonlinear systems. Using the shooting method we can find a number of periodic oscillations with different periods for the same state of system. This phenomenon do not appear in weakly nonlinear systems.

- The calculation of the periodic solutions of Eq. (32) was implemented by a self - written computer program in MATLAB environment. To verify the correctness of the procedure using the shooting method, the procedure using the incremental harmonic balance method is also applied to solve the same problem. The computation time with the shooting procedure is shorter than that with the incremental harmonic balance procedure. By using the shooting method we can find more multiple periodic attractors than by the using the incremental harmonic balance method.

\section{ACKNOWLEDGMENT}

This paper was completed with the financial support by the Vietnam National Foundation for Science and Technology Development (NAFOSTED). 


\section{REFERENCES}

[1] Nayfeh A. H., Balachandran B., Applied Nonlinear Dynamics, John Wiley \& Sons, New York, (1995).

[2] Seydel R., Practical bifurcation and stability analysis, Springer - Verlag, New York, (1994).

[3] Parker T. S., Chua L. O., Practical Numerical Algorithms for Chaotic Systems, Springer Verlag, New York, (1995).

[4] Thai Manh Cau, Calculating nonlinear vibrations of some mechanics models in machinery using MAPLE software, PhD - Thesis, Hanoi University of Science and Technology, (2004) (in Vietnamese).

[5] Hoang Manh Cuong, Calculating periodic vibrations and Bifurcations of some of vibration models in machinery, PhD - Thesis, Hanoi University of Science and Technology, (2011) (in Vietnamese).

[6] Xu L., Lu M. W., Cao Q., Nonlinear vibrations of dynamical systems with a general form of piecewise - linear viscous damping by incremental harmonic balance method, Physics Letters A, 301 (2002) $65-73$.

[7] Wong C. W., Zhang W. S., Lau S. L., Periodic forced vibration of unsymmetrical piecewise - linear systems by incremental harmonic balance method, Journal of Sound and Vibration, 149(1) (1991) 91 - 105.

[8] Raghothama A., Narayaman S., Bifurcation and chaos of an articulated loading platform with piecewise non - linear stiffness using the incremental harmonic balance method, Ocean Engineering, 27 (2000) 1087 - 1107.

[9] Cao Q., et. al., Analysis of period - doubling and chaos of a non - symmetric oscillator with piecewise - linearity, Chaos, Solutions and Fractals, 12 (2001) 1917 - 1927.

[10] Xu L., Lu M. W., Cao Q., Bifurcation and chaos of harmonically excited oscillator with both stiffness and viscous damping piecewise linearities by incremental harmonic balance method, Journal of Sound and Vibration, 264 (2003) 873 - 882.

[11] Müller P. C., Schiehlen W., Lineare Schwingungen, Akademische Verlagsgesellschaft, Wiesbaden, (1976).

[12] Nguyen Van Dao, Stability of Dynamic Systems, Vietnam National University Publishing House, Hanoi, (1998).

[13] Malkin J. G., Theorie der Stabilität einer Bewegung, Akademie, Berlin, (1959).

[14] Nguyen Van Khang, Dynamische Stabilität und periodische Schwingungen in Mechanismen, Diss. B, TH Karl-Marx-Stadt, (1986).

[15] Nguyen Van Khang, Numerische Bestimmung der dynamischen Stabilitätsparameter und periodischen Schwingungen ebener Mechanismen, Rev. Roum. Sci. Tech. Mec. Appl., 27(4) (1982) $495-507$.

[16] Nguyen Van Khang, Nguyen Phong Dien, Hoang Manh Cuong, Linearization and parametric vibration analysis of some applied problems in multibody systems, Multibody System Dynamics , 22(2) (2009) 163 - 180.

[17] Hsu C. S., A generalized theory of cell - to - cell mapping for nonlinear dynamical systems, Journal of Applied Mechanics, 48 (1981) 634 - 642.

[18] Kreuzer E., Numerische Untersuchung nichtlinearer dynamischer Systeme, Springer Verlag, (1987). 